\title{
Anastomotic leakage could be caused by regorafenib - a case report
}

\author{
Tzu-Chi Hsu ${ }^{1,2,3}$, Wen-Chun Sun ${ }^{1}$ and Ming-Jen Chen ${ }^{1,3}$ \\ ${ }^{1}$ Division of Colon and Rectal Surgery, Department of Surgery, Taipei Mackay Memorial Hospital, Taipei, Taiwan \\ ${ }^{2}$ Department of Surgery, Taipei Medical University, Taipei, Taiwan \\ ${ }^{3}$ Department of Surgery, Mackay Medical College, New Taipei, Taiwan
}

\begin{abstract}
A 53 years old female had an extended right hemicolectomy with anastomosis for adenocarcinoma of transverse colon with intestinal obstruction on 2008-2-19. Pathology report revealed a Dukes' C lesion with one out of 22 lymph nodes showing metastasis. Eight courses of adjuvant chemotherapy with capecitabine were given. CEA was Elevated to $11.43 \mathrm{ng} / \mu \mathrm{l}$ on $2011-7-19$. CT scan on $2012-1-16$ revealed "a $1.8 \times 1.5 \mathrm{~cm}$ soft tissue nodule at left anterior pararenal space showing slightly increasing in size as compared to previous CT". After failure of multiple lines of chemotherapy with oxaliplatin, irinotecan, xeloda, tegafur-uracil, leucovorin and biologics of bevacizumab, Erbitux, the patient was started to receive $160 \mathrm{mg}$ /day of regorafenib from 2015-10-13. CT scan on 2015-12-23 was reported as "A $3 \times 3.6 \mathrm{~cm}$ growing metastatic mass with central necrosis in left upper abdomen with adjacent small bowel invasion". Because of progressive enlargement of the metastatic tumor mass, surgical resection of the metastasis (metastasectomy) was suggested. The last dose of regoarfenib was taken on 2015-12-15. During laparotomy, a $4 \times 3.5 \times 3.5 \mathrm{~cm}$ in size tumor mass was located at the left upper abdomen with involvement of several loops of jejunum. Two metastastic tumors were also found between the omentum and abdominal wall. Resection of metastatic sites together with a segment of small bowel and jejunojejunostomy was performed on 105-1-5. She was found to have bile stained discharge from the drain site on 105-1-15. An emergent laparotomy was performed on the same day. Purulent discharge was accumulated at the left upper part of the abdomen near the resected tumor site. Previous jejunojejunostomy site were not able to be visualized because of severe adhesions. No apparent leakage site was able to be seen, however, leakage of anastomosis was strongly suggested. Adequate drainage of intra-abdominal abscess was performed. The patient received postoperative TPN with slowly decrease of the fistula drainage and eventually healing of the anastomosis.
\end{abstract}

\section{Introduction}

Bevacizumab is a humanized monoclonal antibody that targets the VEGF molecule. It is known to be associated with bowel perforation and bowel ischemia [1-4]. Regorafenib, an oral multikinase inhibitor targeting multiple tumor pathways [1-6]. However, incidence of gastrointestinal perforation and thrombotic effect was not available in the clinical studies $[7,8]$. We have experienced a patient with leakage of bowel anastomosis following resection of metastatic sites together with a segment of small bowel and jejunojejunostomy. Emergent laparotomy with adequate drainage of intra-abdominal abscess result in a smooth postoperative course.

\section{Case report}

A 53 years old female had an extended right hemicolecctomy with anastomosis for transverse colon cancer with intestinal obstruction on 2008-2-19. Postoperative course was uneventful. Pathology report revealed a Dukes' C lesion with one out of 22 lymph nodes showing metastasis. Eight courses of adjuvant chemotherapy with capecitabine were given. She was regularly followed at the colorectal service of Taipei Mackay Memorial Hospital. CEA was elevated to $11.43 \mathrm{ng} / \mu \mathrm{l}$ on 20117-19. CT scan on 2011-7-28 was reported as "Post OP status without recurrent tumor seen in anastomatic region". Further elevation of CEA to $12.89 \mathrm{ng} / \mu \mathrm{l}$ was noted on $2011-9-29$ and to $20.83 \mathrm{ng} / \mu \mathrm{l}$ on 2011 12-20. CT scan on 2012-1-16 revealed "A $1.8 \times 1.5 \mathrm{~cm}$ soft tissue nodule at left anterior pararenal space showing slightly increasing in size as compared to previous CT". She had nine courses of tegafur-uracil and leucovorin from 2012-1-21 to 2012-7-14. CT scan on 2013-12-4 was reported as "Relatively stationary appearance of an ill-defined soft tissue density in the left upper abdomen as compared with previous CT scan dated on 2013-8-7. However, changing size of the lesion was noted compare with previous serial CTs, raising concern for omentum seeding" was reported on 2013-11-28 . CT scan on 2014-4-1 was reported as "an about $1.3 \mathrm{~cm}$ irregular lobulated soft tissue nodule at left upper omentum, mild increase in size as compared with previous CT on 2013-12-4, R/O omental seeding." She had 14 courses of irinotecan, tegafur-uracil, leucovorin and bevacizumab from 2012-7-21 to 2013$2-21$, then eight courses of oxaliplatin, tegafur-uracil and leucovorin from 2013-3-7 to 2013-9-5, followed by eight courses of irinotecan, capecitabine and cetuximub from 2013-9-26 to 2014-3-20. She was then treated with 28 courses of irinotecan and capecitabine following failure of above regimens of chemotherapy and biologics.

CT scan on 2014-7-17 was reported as "a growing nodule at left upper omentum, mild increase in size as compared with previous CT on 2014-4-1 (from $1.3 \mathrm{~cm}$ to $2.0 \mathrm{~cm}$ ), suggesting omental seeding." CT scan on 2014-11-8 was reported as "Increasing in size of the left upper omental nodule (from $2 \mathrm{~cm}$ to $2.4 \mathrm{~cm}$ in diameter) as compared to last CT dated on 2014-7-17, suggest clinical correlation." CT scan on 2015-

*Correspondence to: Tzu-Chi Hsu, MD, FACS, FACRS, Division of Colon and Rectal Surgery, Department of Surgery, Taipei Mackay Memorial Hospital, Taipei, Taiwan, Email: tzuchi@mmh.org.tw

Key words: adenocarcinoma, bevacizumab, metastasectomy, regorafenib

Received: July 16, 2018; Accepted: July 23, 2018; Published: July 25, 2018 
2-24 was reported as "still a $2.4 \mathrm{~cm}$ necrotic omtenal nodule in left upper abdomen". CT scan on 2015-9-8 was reported as "Increasing size of the omental nodular lesion was seen comparing to previous CT study."

After failure of multiple lines of chemotherapy and biologics, the patient was started to receive $160 \mathrm{mg}$ /day of regorafenib from 2015-1013 , then decrease to $120 \mathrm{mg}$ /day and eventually to $80 \mathrm{mg} /$ day because of elevated liver enzyme. CT scan on 2015-12-23 was reported as "a $3 \times 3.6 \mathrm{~cm}$ growing metastatic mass with central necrosis in left upper abdomen with adjacent small bowel invasion" (Figure 1).

Because of progressive enlargement of the metastatic tumor mass, surgical resection of the metastasis (metastasectomy) was suggested. The last dose of regoarfenib was taken on 2015-12-15. During laparotomy, a $4 \times 3.5 \times 3.5 \mathrm{~cm}$ in size tumor mass was located at the left upper abdomen with involvement of several loops of jejunum. Two metastastic tumors were also found between the omentum and abdominal wall. Resection of metastatic sites together with a segment of small bowel and jejunojejunostomy was performed on 105-1-5 (Figure 2). She was found to have bile stained discharge from the drain site on 105-1-15. An emergent laparotomy was performed on the same day. Purulent discharge was accumulated at the left upper part of the abdomen near the resected tumor site. Previous jejunojejunostomy site was not able to

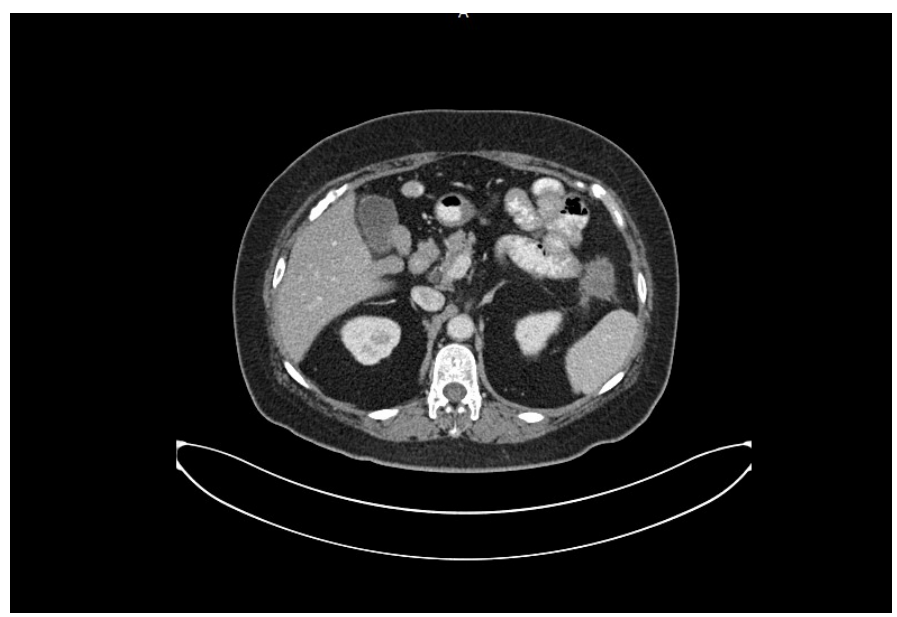

Figure 1. A $4 \times 3.5 \mathrm{~cm}$ necrotic omental nodule in left upper abdomen, with increasing size of the mental nodular lesion comparing to previous CT study

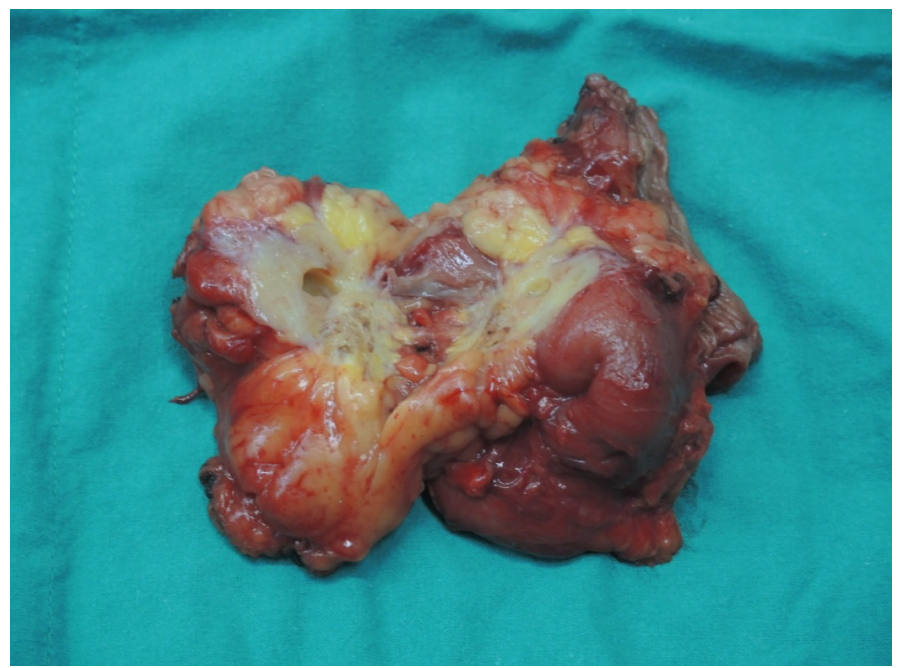

Figure 2. Gross specimen of the resected metastatic tumor be visualized because of severe adhesions. No apparent leakage site was able to be seen, however, leakage of anastomosis was strongly suggested. Adequate drainage of intra-abdominal abscess was performed. The patient received postoperative TPN with slowly decrease of the fistula drainage and eventually healing of the anastomosis.

\section{Discussion}

VEGF is a key mediator of angiogenesis in normal tissues and binds two VEGF receptors (VEGF receptor-1 and VEGF receptor-2), which are expressed on vascular endothelial cells [6]. VEGF is also thought to be a key mediator of angiogenesis in cancer $[1,5]$. Bevacizumab is a humanized monoclonal antibody that targets the VEGF molecule. It is hypothesized that bevacizumab works by both depriving tumors of the neovascularity they require to grow and sustain beyond a size of approximately $2 \mathrm{~mm}$ and by improving local delivery of chemotherapy through alterations of tumor vasculature permeability and Starling forces [1-9]. Although it is not effective as a stand-alone agent, clinical trials have demonstrated the ability of bevacizumab to enhance the effectiveness of chemotherapy for the treatment of metastatic colorectal cancer [1-10]. The most serious adverse events associated with bevacizumab include bowel ischemia, gastrointestinal perforation, wound healing complications, hemorrhage, and arterial thromboembolic events [1-4]. The occurrence of gastrointestinal complications relating to bowel ischemia prompted the issuance of a warning letter addressed to physicians by the manufacturer, and changes in the FDA product labeling to reflect these risks $[11,12]$. They postulated an increased risk for bowel complications in previously irradiated patients and suggested that radiation induced tissue damage may enhance the risk of arterial and venous thromboses associated with bevacizumab [13]. They postulated micro thromboses as the cause of the ischemia [14]. Given the known effects of radiation therapy on microvasculature, including endothelial damage and obliterative fibrosis, it seems plausible that the combination of radiation therapy and bevacizumab treatment may especially predispose to the development of tissue ischemia; In the gastrointestinal tract, the effects of ischemia can be particularly dramatic and catastrophic [13-16]. It is generally recommended that surgery not be undertaken for at least 4-8 weeks following cessation of bevacizumab treatment because of its known inhibitory effects on wound healing $[4,17]$. Wound healing is the result of a sequence of several basic processes including inflammation, cell proliferation, matrix formation and remodeling, angiogenesis, wound contraction, and epithelialization [16].

Regorafenib (BAY 73-4506), an oral multikinase inhibitor targeting multiple tumor pathways1-3. It showed Inhibition of proliferation of tumor cell through biochemical activity of KIT, PDGFR, RET; Inhibition of tumor microenvironment signaling through biochemical activity of PDGFR- $\beta$, FGFR; Inhibition of neoangiogenesis is through biochemical activity of VEGFR1-3, TIE2 $[18,19]$.

A CORRECT study was designed to patients with metastatic colorectal cancer treated with regorafenib or placebo after failure of standard therapy. The result showed that OS rates were consistently higher in the regorafenib arm than in the placebo arm at 6 and 12 months post-randomization. Treatment with regorafenib resulted in a $21 \%$ reduction in the risk of death over placebo. Regorafenib + BSC significantly improved progression-free survival (PFS) and time to progression over placebo + BSC. Treatment with regorafenib resulted in a $50.6 \%$ reduction in the risk of progression or death over placebo [7]. Frequently seen adverse event of regorafenib include hand-foot skin reaction, fatigue, hypertension, diarrhea, and rash/desquamation. 
However, incidence of gastrointestinal perforation and thrombotic effect was not available in the clinical studies $[7,8]$. It is understandable that not many patients would have chance of major surgery following usage of regorafenib since regorafenib is a salvage therapy for the patients with colorectal cancer after multiple lines of chemotherapy. Surely the problem of bowel anastomosis would be very rare because the chance of bowel resection would be very uncommon. However, since regorafenib also have biochemical activity of VEGFR1-3, rational thought would be it might affect bowel blood supply in some extent. Stopping of regorafenib prior to major surgery such as laparotomy and bowel resection with anastomosis might be the best for the patient's interest. At the moment, we do not know how long ahead should the regorafenib be stopped before such event. This case had regorafenib stopped 20 days before laparotomy but still had an anastomotic leakage. Of course, the cause of anastomotic leakage is many. Factors affecting anastomotic healing include poor surgical technique, wrong intraoperative judgement, local complications (sepsis, bowel preparation, drains, role of omentum and peritoneum, anaesthetic drugs, protective stoma), systemic complications (nutritional status, blood loss) and surgeon-related factors [20]. However, prior usage of biologic such as regorafenib still needed to be considered. It might not be necessary like patients who are receiving bevacizumab therapy to have biologic stopped 4-6 weeks prior to surgery. However, stop regorafenib for period of time prior to surgery and major bowel resection should always be thought and carried out to avoid catastrophic complication such as an anastomotic leakage.

\section{References}

1. Hurwitz H, Kabbinavar F (2005) Bevacizumab combined with standard fluoropyrimidine-based chemotherapy regimens to treat colorectal cancer. Oncology 69: 17-24.

2. Saif MW, Elfiky A, Salem RR (2007) Gastrointestinal perforation due to bevacizumab in colorectal cancer. Ann Surg Oncol 14: 1860-1869. [Crossref]

3. Genentech I (2007) VEGF, Angiogenesis and Cancer.

4. Saif MW, Mehra R (2006) Incidence and management of bevacizumab-related toxicities in colorectal cancer. Expert Opin Drug Safe 5: 553-566. [Crossref]

5. Carmeliet P (2005) VEGF as a key mediator of angiogenesis in cancer. Oncology 69 Suppl 3: 4-10. [Crossref]
6. Thornton AD, Ravn P, Winslet M, Chester K (2006) Angiogenesis inhibition with bevacizumab and the surgical management of colorectal cancer. Br J Surg 93: 14561463. [Crossref]

7. Grothey A, Van Cutsem E, Sobrero A, (2013) Regorafenib monotherapy for previously treated metastatic colorectal cancer (CORRECT): an international, multicentre, randomised, placebo-controlled, phase 3 trial. Lancet 381: 303-312.

8. Li J, Qin S, Xu R (2015) Regorafenib plus best supportive care versus placebo plus best supportive care in Asian patients with previously treated metastatic colorectal cancer (CONCUR): a randomised, double-blind, placebo-controlled, phase 3 trial. Lancet Oncology 16: 619-629.

9. Verhoef C, de Wilt JH, Verheul HM (2006) Angiogenesis inhibitors: Perspectives for medical, surgical and radiation oncology. Curr Pharm Des 12: 2623-2630.

10. Kabbinavar F, Hurwitz HI, Fehrenbacher L (2003) Phase II, randomized trial comparing bevacizumab plus fluorouracil (FU)/ leucovorin (LV) with FU/LV alone in patients with metastatic colorectal cancer. J Clin Oncol 21: 60-65.

11. Ratner M (2004) Genentech discloses safety concerns over Avastin. Nat Biotechnol 22: 1198. [Crossref]

12. Biotechnology, G: AVASTIN (bevacizumab) Package Insert. 2006

13. Lordick F, Geinitz H, Theisen J, (2006) Increased risk of ischemic bowel complications during treatment with bevacizumab after pelvic irradiation: Report of three cases. Int $J$ Radiat Oncol Biol Phys 64: 1295-1298.

14. Heinzerling JH, Huerta S (2006) Bowel perforation from bevacizumab for the treatment of metastatic colon cancer: Incidence, etiology, and management. Curr Surg 63: 334 337.

15. Wang J, Boerma M, Fu Q (2007) Significance of endothelial dysfunction in the pathogenesis of early and delayed radiation enteropathy. World J Gastroenterol 13: 3047-3055.

16. Wang J, Boerma M, Fu Q (2006) Radiation responses in skin and connective tissues: Effect on wound healing and surgical outcome. Hernia 10: 502-506.

17. Scappaticci FA, Fehrenbacher L, Cartwright T (2005) Surgical wound healing complications in metastatic colorectal cancer patients treated with bevacizumab. J Surg Oncol 91: 173-180.

18. Wilhelm SM, Dumas J, Adnane L (2011) Regorafenib (BAY 73-4506): a new ora multikinase inhibitor of angiogenic, stromal and oncogenic receptor tyrosine kinases with potent preclinical antitumor activity. Int J Cancer 129: 245-55.

19. Strumberg D, Schultheis B (2012) Regorafenib for cancer. Expert Opin Investig Drugs 21: 879-889. [Crossref]

20. Colorectal anastomotic leakage (2013) Aspects of prevention, detection and treatment World J Gastroenterol 19: 2293-2297.

Copyright: (C2018 Tzu-Chi Hsu. This is an open-access article distributed under the terms of the Creative Commons Attribution License, which permits unrestricted use, distribution, and reproduction in any medium, provided the original author and source are credited. 